\title{
CRÓNICA PARLAMENTARIA DEL SENADO
}

"Tienen los gobiernos datos y elementos de juicio que a ellos, principalmente incumbe apreciar, para la orientación de la política; si no merecen confianza hay que barrerlos, y si merecen confianza hay que otorgársela, a reserva del inalienable derecho de crítica en la hora del fracaso"

(Jose Canalejas: La política liberal en España, 1912)

La presente crónica parlamentaria abarca el periodo de sesiones que se proyecta desde julio de 1986 al 23 de diciembre del mismo año, configurando el primer periodo de sesiones de la Cámara Alta de la III Legislatura Constitucional, así como el periodo de sesiones que se prolonga de febrero a junio de 1987.

La sesión celebrada el día 15 de julio de 1987 marcaría la reanudación de actividades del Pleno del Senado que estaría configurada por una serie de pasos que, por su trascendencia en el orden de práctica parlamentaria, conviene, cuando menos, reseñar.

Desde la publicación del Real Decreto 794/1986 de 22 de abril, de disolución del Congreso de Diputados y del Senado y de convocatoria de elecciones, hasta la Junta preparatoria del Senado que se celebraría el martes 15 de julio de 1986, sería la Diputación permanente del Senado la que asumiría, tal y como prevé la Constitución en su articulo 78-1, 2 y 3, la actividad del Pleno del Senado.

\section{PRÁCTICAS APLICATIVAS DE LA CONSTITUCIÓN}

Las reflejadas en el presente periodo de sesiones derivan principalmente de la disolución de las Cortes Generales por iniciativa del Presidente de Gobierno, conforme al artículo 115-1 de la Constitución.

\section{A Prácticas de carácter no legislativo}

Dentro de este ámbito, podemos referirnos a las legislaturas siguientes que se llevarian a cabo en la sesión celebrada por la Junta Preparatoria 
de 15 de julio de 1986, de la tercera legislativa y que queda reflejada en el Diario de Sesiones del Senado número primero. (DSS n. ${ }^{\circ}$; 15 de julio de 1986 - III Legislatura.)

1.A.a. Apertura de la Cámara. (DSS n. ${ }^{\circ}$, martes 15 de julio de 1986 - III Legislatura.)

\section{A.a.1. Lectura del Real Decreto de Convocatoria de la Junta}

Preparatoria y de la lista de Senadores electos, por el orden de presentación de sus credenciales. (DSS n. ${ }^{\circ}$, 15 de julio de 1986.)

El Presidente de la Junta Preparatoria, por figurar en primer lugar en la lista de presentación de credenciales, tal y como dispone el artículo 2-2 del Reglamento del Senado, procedió al acto reglamentario de celebración de la Junta Preparatoria, trámite legal éste, previo —tal como prescribe el Reglamento del Senado, artículo 3,- a la Constitución de la Mesa.

Una vez configurada la Presidencia de la Junta Prepatatoria, y en cumplimiento del artículo 2-2 R.S. mencionado con anterioridad, fue declarada abierta la sesión, y se procedió a la lectura de la Convocatoria de la Cámara por parte del Letrado Mayor del Senado, Sr. Serrano Alberca, así como a la lista de Senadores, por orden de presentación de credenciales y los artículos del Reglamento referentes a dicho acto.

Leída la relación de los 241 Senadores, se procedió a la Designación de la Mesa de Edad.

1.A.a.2. Designación de la Mesa de Edad y Constitución definitiva de la Cámara.

Conforme al artículo 3 del R.S., se formaría la Mesa de Edad, correspondiendo la Presidencia de la misma al Senador Calvo López, recayendo, asimismo, la condición de Secretarios a la misma a los cuatro Senadores más jóvenes: Sr. Granado Martínez, Cerdeira Monterero, Santiago Lavado y Martínez Guijarro.

Una vez integrada la Mesa de Edad y a instancias del Presidente de la misma, Sr. Calvo López, se procedió a la Constitución de la Mesa del Senado, al no mediar obstáculo alguno para ello, ya que los recursos contenciosos-electorales contra la proclamación de Senadores de elección directa no alcanzaron el 20 por 100 de los escaños que integran el Senado, según el apartado $1 \mathrm{del}$ artículo cuarto del Reglamento del Senado. 
La Constitución definitiva de la Mesa, que se efectuaria, tal y como prescribe el artículo 66 R.S., por votación a través de papeletas, quedó perfilada del siguiente tenor:

Presidente: Sr. Carvajal Pérez, que obtendría 235 votos a favor, y seis en blanco.

Vicepresidente: Sr. Rodríguez Pardo, con 147 votos a favor.

Vicepresidente: Sr. Arespacochaga, con 76 votos y 15 blancos.

Secretario primero: Sra. Urcelay López de las Heras; 132 votos.

Secretario segundo: Sr. Díez González; con 118 votos.

Secretario tercero: Sr. Aguilera Bermúdez, con 68 votos.

Secretario cuarto: Sr. Gamunide Alix, con 58 votos.

Tras el juramento o promesa, tal y como define el art. 11 del R.S., la Mesa del Senado, ya constituída, así como por los Senadores, de acatamiento de la Constitución quedaría definitivamente constituido el Senado. La Presidencia lo comunicaria oficialmente al Rey, al Congreso de Diputados y al Gobierno así como a las Asambleas de las Comunidades Autónomas de conformidad con el artículo 14 del Reglamento del Senado.

\section{A.a.3. Lectura del Informe aprobado por la Diputación permanente.}

El Letrado Mayor, Sr. Serrano Alberca, en cumplimiento con lo previsto constitucionalmente en que la Diputación permanente ha de informar a la Cámara recién constituida, procedió a dar lectura al Informe aprobado por aquélla, y que afectaría al funcionamiento y actividad de la Diputación Permanente del Senado, una vez que fueron disueltas las Cortes Generales por Real Decreto de la Jefaicra del Estado, publicado en el BOE el 23 de abril de 1986. En tal sentido, el Informe aludía al cumplimiento por la Diputación Permanente, del mandato constitucional del artículo 78-2 de la Norma Suprema, «Velando fielmente por los poderes de la Cámara al no encontrarse reunida. La Diputación Permanente, a través de la Presidencia, en la que al efecto delegó, ha resuelto asimismo los asuntos de la administración ordinaria sometidos a su consideración».

Con la nueva Constitución de la Cámara, quedaría de nuevo pendiente la elección de los miembros de la nueva diputación Permanente. 
1.A.b. Elección los miembros de la Diputación Permanente del Senado (DSS n. ${ }^{\circ} 2$, martes 29 julio de 1986 - III Legislatura).

Al amparo del artículo 45 del R. S. que establece que tan pronto se constituya definitivamente la Cámara, procederá a designar la Diputación Permanente, presidida por el Presidente del Senado e integrada por un mínimo de 21 miembros, en proporción al número de componentes de los respectivos grupos Parlamentarios, a propuesta de la Comisión.

Siendo competencia del Pleno la determinación del número de miembros de la Diputación Permanente la Junta de Portavoces acordó proponer a la Cámara que continuara el mismo número de la Legislatura anterior, esto es 27 senadores y 27 suplentes. Dicha designación se distriburia de la manera siguiente:

- Al grupo socialista, sin contar con la Presidencia, le corresponden 15,3 Senadores.

- Al grupo Coalición Popular - 6,6 senadores.

- Al grupo Convergencia y Unió - 1,1 Senadores.

- Al grupo Senadores Nacionalistas Vascos -1 Senador.

- Al grupo Mixto, 1,8 Senadores.

Lo que a efectos prácticos, se traduciría en lo siguiente:

- Grupo Socialista: 15 Senadores.

- Grupo Coalición Popular: 7 Senadores.

- Grupo Convergencia y Unió: 1 Senador.

- Grupo Senadores Nacionalistas Vascos: 1 Senador.

- Grupo Mixto: 2 Senadores. 
1.A.C. Presentación del Gobierno (DSS, n. ${ }^{\circ}$, miércoles, 1 octubre de 1986 - III Legislatura).

Especial mención merece lo que puede considerarse, de seguir produciéndose, una exquisita práctica de cortesía institucional, como lo es la presentación del Gobierno a la Alta Cámara.

Asimismo lo entenderia el Presidente de Gobierno, al referirse en el mencionado acto, a la repetición de la presentación del Gobierno que en los mismos términos llevó a cabo en 1982 también ante el Senado.

El Presidente de Gobierno se referiría al hecho incontestable de que esta práctica no obedece a ningún mandato constitucional, sino «al sentimiento, muy expresivo, de su voluntad democrática de presentar, ante el Senado, al Gobierno de la Nación", aludiendo a que no se habia hecho con anterioridad, desde principios de siglo, recuperándose así una cortés tradición.

Sería en cierto modo esta práctica como la correlación de lo que ante el Congreso - y por explícito mandato constitucional-corresponderia al debate de investidura, mudando lo mudable.

La intervención del Presidente del Gobierno no exenta de alusiones a determinados temas tratados, con motivo de la presentación de su programa para conseguir su investidura, incidió en algunos aspectos que a continuación transcribimos:

En primer lugar, recalcó la importante función que la Cámara Alta ejercita en aras de la profundización y de fortalecimiento del sistema autónómico, de un lado, y de segunda lectura y reflexión sobre las actuaciónes del Congreso. Destacando su papel relevante como paso político, donde los representantes populares deben manifestar las aspiraciones y puntos de vista de los electores, así como via para que cada fuerza política o cada Comunidad Autónoma, se vaya definiendo oficialmente sobre temas específicos. Subrayó de igual modo la potenciación de esta segunda cámara en orden a su papel coadyuvador de la formación de la opinión, alejando de la misma el papel pasivo al que, frecuentemente, parece que quieren reducirla algunos sectores.

El relevante papel de la Cámara Alta, justifica por sí mismo -aduciría el Presidente del Gobierno- su presencia para presentar el gobierno, como símbolo de la fluida cooperación institucional que es de desear.

La intervención del Presidente del Gobierno fue seguida de la de los portavoces de los grupos parlamentarios. En este sentido, nos referimos a continuación muy brevemente a cada una de éstas. 
En contestación a tales intervenciones, el Presidente de Gobierno subrayó la naturaleza del acto de presentación del Gobierno y no un planteamiento de debate de cuestiones de carácter general, como tampoco de cuestiones de carácter específico. Por ello insistió en que su respuesta debería interpretarse en un estricto sentido de cortesía parlamentaria frente a cada uno de los intervinientes, y de respeto a la propia Cámara Alta.

Habiendo, pues, dejado sentado que en ningún caso podía calificarse su intervención como un debate - por no estar así previsto en los términos de la comparecencia - apuntó una reflexión de caracter general, sin perjuicio de dar respuesta con posterioridad a cada una de las intervenciones de los senadores.

\section{B. Prácticas de caracter legislativo}

En los dos períodos de sesiones que venimos reseñando, no podemos mencionar prácticas de tal indole, pues sin perjuicio de que las haya habido en el Congreso, dado que nuestro estudio está referido a la Cámara del Senado, éste no puede pronunciarse, como es sabido y por imperativo del artículo 86-2 de la Constitución, sobre su hipotética convalidación.

\section{ACTIVIDAD LEGISLATIVA}

Hemos ordenado la actividad legislativa que se produjo, atendiendo a la división de la misma en los dos períodos de sesiones que venimos tratando; de igual modo, tal y como viene siendo habitual, distinguiremos entre la actividad legislativa que deriva de la iniciativa gubernamental (proyectos de ley), y la de procedencia de las Cortes Generales (proposiciones de Ley).

En este sentido reflejamos a continuación, en primer término, la actividad legislativa que se ha producido en la Cámara Alta durante el período de Sesiones primero de la III Legislatura: octubre - diciembre 1986, comprendiendo tanto los proyectos de Ley como las proposiciones de Ley; y en segundo lugar, la actividad legislativa asumida por el Senado a lo largo del segundo período de sesiones: febrero - junio de 1987, comprendiendo asimismo tanto los proyectos de Ley como las proposiciones de Ley. 


\section{A. Actividad legislativa}

Período de sesiones octubre-diciembre 1986.

\section{A.1. Proyectos de Ley}

Proyecto de Ley Orgánica por el que se autoriza la ratificación por España del Acta Unica Europea, firmada en Luxemburgo el 17 de febrero de 1986 (BOCG, Senado Serie II, número 1, del 10 de octubre de 1986) y (DSS, número 6, 29 octubre del 1986).

Proyecto de Ley por el que se conceden determinadas exenciones fiscales y aduaneras al Instituto de Relaciones Europeo - Latino americano (IKELA) (BOCG, Senado, Serie II, n. ${ }^{\circ}$, del 23 de octubre de 1986) y (DSS, n. ${ }^{\circ} 11$ de 3 de diciembre de 1986).

Dictamen de la Comisión de Presupuestos en relación con el Proyecto de Ley de Presupuestos Generales del Estado para 1987. Tramitado por el procedimiento de Urgencia (BOCG, Senado, Serie II, n. ${ }^{\circ} 7$ de 13 de diciembre de 1986) y (DSS, n. ${ }^{\circ} 12-13-14-15$ y 16 del día 15-16-17-18-19 de diciembre de 1986).

Dictamen de la Comisión de Defensa en relación con el proyecto de Ley de rehabilitación de militares profesionales. Se tramita por el procedimiento de urgencia. (BOCG, Senado, Serie $.11, n .{ }^{\circ} 8$ de 13 de diciembre de 1986) y (DSS, . $^{\circ} 16$ de fecha 19 de diciembre de 1986).

Dictamen de la Comisión de Justicia en relación con el proyecto de Ley de supresión de las tasas judiciales. (BOCG, Senado, Serie II, n. ${ }^{\circ} 3$, de 10 diciembre 1986.) y (DSS, $n .^{\circ} 16$ de fecha 19 diciembre de 1986.)

Dictamen de la Comisión de Agricultura y Pesca, en relación con el proyecto de Ley por el que se establecen las Bases del Régimen Jurídico de las Cámaras Agrarias. (BOCG, Senado, Serie II, $n .^{\circ} 5$ de 12 diciembre de 1986.) (DSS, n. ${ }^{\circ} 17$ de 20 de diciembre de 1986.)

\section{A.2. Proposiciones de Ley}

Proposiciones de Ley de regulación de beneficios a los fàmiliares. (BOCG, Senado, Serie II, n. ${ }^{\circ} 4$, de 12 de noviembre de 1986.) y (DSS, n. ${ }^{\circ} 11$ de 3 de diciembre de 1986). 


\section{B. Actividad Legislativa}

Período de Sesiones febrero-junio 1987.

\section{B.1. Proyectos de Ley}

Dictamen de la Comisión de Economía y Hacienda en relación con el proyecto de Ley sobre Fiscalidad municipal en la ordenación del tráfico urbano (DSS, n. ${ }^{\circ} 21$ de 4 marzo de 1987).

Dictamen de la Comisión de trabajo en relación con el proyecto de Ley General de Cooperativas (DSS, n. ${ }^{\circ} 21$ de 4 de marzo de 1987).

Dictamen de la Comisión de Constitución, en relación con el Proyecto de Ley Orgánica que modifica la Ley Orgánica 5/1985 de 19 junio de Régimen Electoral General, que regula las elecciones al Parlamento Europeo. Se tramita por procedimiento de urgencia (DSS, $n .^{\circ} / 25$ de 31 de marzo de 1987).

Dictamen de la Comisión de Constitución de relación con el Proyecto de Ley Orgánica de Conflictos jurisdiccionales (DSS, n. 25 de fecha 31 marzo 1987).

Proyecto de Ley por el que se autoriza la participación de España en el Fondo Especial para el Africa Sub-Sahariana (DSS, $n .^{\circ} 28$ de 28 de abril de 1987).

Proyecto de Ley sobre concesión de un crédito extraordinario, por importe total de 1.997.125.969 ptas para abono de pensiones asistenciales a ancianos y enfermos incapacitados, por diferencias producidas en el año 1985 (DSS, n. ${ }^{\circ} 28$ de abril de 1987).

Dictamen de la Comisión de Asuntos exteriores en relación con el proyecto de Ley de tasas consulares (DSS, $\mathrm{n} .{ }^{\circ} 28$ de 28 de abril de 1987).

Dictamen de la Comisión en relación con Proyecto de Ley sobre dotaciones presupuestarias para inversiones y sostenimiento de las Fuerzas Armadas (DSS, $n .^{\circ} 28$ de abril de 1987).

Dictamen de la Comisión de presidencia del Gobierno e Interior en relación con el proyecto de Ley de órganos de representación, determinación de las condiciones de trabajo y participación del personal al servicio de las Administraciones públicas (DSS, $n .^{\circ} 32$ de 19 de marzo de 1987). 
Dictamen de la Comisión de Economía y Hacienda en relación con el Proyecto de Ley de regulación de los planes y fondos de pensiones (DSS, n. ${ }^{\circ} 33$ de fecha 20 de marzo de 1987).

Dictamen de la Comisión de Industria y Energía, Comercio y Turismo, en relación con Proyecto de Ley de disposiciones básicas para el desarrollo combinado en materia de combustibles gaseosos (DSS, n. ${ }^{\circ} 34$ de fecha 21 mayo de 1987).

Conocimiento directo del pleno referente al proyecto de Ley por el que se autoriza la ampliación de la dotación del tesoro al crédito oficial durante 1987 para la concesión de crédito financiero a Bolivia (DSS, n. ${ }^{\circ} 37$ de 17 de junio de 1987).

Dictamen de la Comisión de Educación, Universidades, Investigación en relación con el Proyecto de Ley sobre gratuidad de los estudios de Bachillerato, Formación Profesional y Artes aplicadas y Oficios Artísticos (DSS, n. ${ }^{\circ} 38$ de 16 junio 1987).

Dictamen de la Comisión de Justicia sobre el Proyecto de Ley Orgánica de la Competencia y organización de la jurisdicción militar (DSS, n. ${ }^{\circ} 38$ de 23 de junio de 1987).

\section{B.2. Proposiciones de Ley}

Toma en consideración de la proposición de Ley Orgánica por parte del Senado, por la que se adiciona un nuevo párrafo a la Disposición transitoria trigesimo-cuarta de la Ley Orgánica 6/1985 de 1 de julio del Poder Judicial. (BOCG, Senado, Serie II, $n .{ }^{\circ} 6$, de fecha 20 noviembre de 1986) y (DSS, n. ${ }^{\circ} 19$, de 10 febrero de 1987).

Toma en consideración de la proposición de Ley de reforma de la Ley de propiedad horizontal procedentes del Senado (DSS, $\mathrm{n} .{ }^{\circ} 30$ de fecha 12 de mayo de 1987).

Dictamen de la Comisión en relación con la proposición de Ley Orgánica sobre financiación de los partidos políticos (DSS, . $^{\circ} 36$ de 16 junio 1987).

Proposición de Ley sobre acceso a la propiedad de los Contratos de arrendamiento rústicos prorrogados en virtud de la Ley 1/1987 de 12 de febrero (DSS, $\mathrm{n} .^{\circ} 37$ de 17 junio de 1987). 


\section{C. Tratados y convenios internacionales}

En el primer período de sesiones de la presente Legislatura, no fue registrado ninguno en la Cámara del Senado, no así en el segundo periodo de sesiones - de febrero a junio de 1987- del que daremos, a continuación una breve reseña. Algunos de los cuales serán citados de forma agrupada cuando estén reflejados en el mismo Diario de Sesiones.

Protocolo de Defensa Aérea entre el Reino de España y la República Francesa. (BOCG, Senado, Serie II, n. ${ }^{\circ} 9$ del 11 de diciembre de 1986.) (DSS, n. ${ }^{\circ} 19$ de fecha 10 febrero de 1987.)

El Senador Barreiro Gil, encargado de la defensa del mismo, renunciaría a la misma por considerar la exposición de motivos suficientes. La votación daría el resultado de 156 votos a favor, uno en contra y 27 abstenciones de un total de 184 votos emitidos, lo que implicaría la autorización al Gobierno para prestar su consentimiento para obligarse por medio del Protócolo de defensa aérea entre el Reino de España y la República Francesa.

Convención sobre la protección física de los materiales nucleares, hecha en Viena y Nueva York, el dia 3 de marzo de 1980, y reserva de la misma (DSS, n. ${ }^{\circ} 21$ de 4 marzo 1987).

Acuerdo Complementario del Convenio Básico de Cooperación Científica y Técnica Hispano - Colombiano, entre el Gobierno de la República de Colombia y el Gobierno del Reino de España, para el desarrollo de un programa en materia sociolaboral, hecho en Bogotá, el 19 de diciembre de 1985. (DSS, n. ${ }^{\circ} 21$ de 4 marzo 1987.)

Seis acuerdos de Cooperación Técnica con diferentes países de Sudamérica y Centroamérica. (DSS, $n .^{\circ} 21$ de 4 de marzo de 1987.)

Canje de Notas Hispano Alemán, ampliando el alcance del Convenio Europeo n. ${ }^{\circ}$ 24, de extradición, de 13 de diciembre de 1987, de fechas: 11 de marzo de 1986 la Nota alemana y de 14 marzo de 1986, la Nota Española, firmadas en Bonn. (DSS, $\mathrm{n} .^{\circ} 21$ de marzo 1987.)

Convenio de la Haya $n .^{\circ} \mathrm{X}$, sobre Competencia de las autoridades y la Ley aplicable en materia de protección de menores, hecho en La Haya el 5 de Octubre de 1961. (DSS, n. ${ }^{\circ} 21$ de 4 marzo 1987.)

Convenio entre España y Luxemburgo para evitar la doble imposición en materia de Impuestos sobre la Renta y Patrimonio y para prevenir el fraude y la evasión fiscal y protocolo anejo, hecho en Madrid el 3 de junio de 1986. (DSS, n. 21 de 4 marzo 1987.) Se aprobará sin discusión. 
Convenio sobre elaboración de una farmacopea europea, hecho en Estrasburgo el 22 de julio de 1964. (DSS, $n .^{\circ} 21$ de igual fecha 4 marzo de 1987.)

Convenio sobre Cooperación Internacional en materia de asistencia administrativa a los refugiados, hecho en Roma el 6 de septiembre de 1984. (DSS, n. ${ }^{\circ} 21$ de 4 marzo 1987.)

Convenio n. $^{\circ}$ XIV de la Conferencia de La Haya de Derecho Internacional privado relativo a la notificación o traslado én el extranjero de documentos judiciales y extrajudiciales en materia civil o comercial hecho en La Haya el 15 de noviembre de 1965 . (DSS, ${ }^{\circ}{ }^{\circ} 21$ de 4 marzo 1985.)

Todos los reseñados fueron aprobados sin discusión. Asimismo fueron sometidos a conocimiento por el pleno del Senado los siguientes Tratados y Convenios internacionales remitidos por el Congreso de los Diputados, y respecto a los que pudo pronunciarse la Cámara Alta en la sesión plenaria de fecha 31 de marzo de 1987.

Acuerdo para la creación de un fondo de Contrapartida de ayuda alimentaría entre España y la República popular de Mozambique. (DSS, n. ${ }^{\circ}$ 25 de 31 marzo 1987.) El Senador del Partido Demócrata Popular, Sr. García Royo, haciendo uso de un turno a favor explicaría la razón por la que su grupo votaría afirmativamente dicho acuerdo. No habiendo turnos en contra, se llevò a cabo la votación, que arrojará el siguiente resultado: Votos emitidos 172, a favor 171, abstenciones; una.

Acuerdo Hispano-Senegalés para cooperación en materia de energía solar. (DSS, n. ${ }^{\circ} 25$ de 31 de marzo 1987) La votación fue de 175 votos a favor de los 175 emitidos.

Convenio $\mathrm{n} .^{\circ} \mathrm{XXIII}$ de la Conferencia de La Haya de Derecho Internacional privado, sobre conocimiento y ejecución de las resoluciones relativas a obligaciones alimentarias. EI DSS n. 25 de 31 de marzo 1987, refleja el asentamiento máximo en la votación efectuada.

Por su parte, en la sesión plenaria efectuada el 28 de abril de 1987 (DSS, n. ${ }^{\circ} 28$ ) se aprobarian diez Convenios, cuatro Acuerdos y un Tratado; de alguno de los cuales damos referencia a continuación:

- Convenio básido de Cooperación Científica y Técnica entre el Reino de España y la República Popular China.

-Acuerdo de Cooperación en materia de defensa entre el Gobierno del Reino de España y el Gobierno del Reino de Bélgica. 
- Convenio Europeo sobre violencia e irrupciones de espectadores con motivo de manifestaciones deportivas.

-Convenio n. ${ }^{\circ} 5$ de la Comisión Internacional del Estado Civil, sobre la extensión de competencia de los funcionarios para autorizar el reconocimiento de hijos no matrimoniales. Aprobado por 176 votos y 1 abstención.

-Tratado entre España y Perú relativo a transferencia de personas a penas privativas de libertad asi como de menores bajo tratamiento especial. Se aprobaria por 180 votos a favor.

-Acuerdo de Cooperación, entre el Gobierno de España y de Bélgica, en el ámbito de Defensa, 175 votos a favor y una abstención.

Acaso uno de los tratados de mayor transcendencia lo configura el que autoriza al Gobierno a prestar su consentimiento para obligarse a la prohibición de emplazar armas nucleares y otras armas de destrucción en masa, en los fondos marinos y oceánicos y su subsuelo. (DSS, n. ${ }^{\circ} 30$ de 12 de mayo 1987.)

El conocimiento, en último término, de Tratados y Convenios por parte del Senado, en la sesión plenaria $n .^{\circ} 37$ de 17 de junio de 1987, vendria marcada por el signo de la autorización de muchos de aquellos, en materia de Defensa:

-Acuerdo de la OTAN, sobre la comunicación de información técnica con fines de defensa.

-Convenio entre los Estados Parte en el Tratado del Atlántico Norte sobre el Estatuto de sus Fuerzas.

-Convenio sobre Estatuto de la organización del Atlántico Norte de los representantes nacionales y del personal internacional.

-Acuerdo de la OTAN para salvaguardia mutua del secreto de invenciones relativas a la defensa respecto de las cuales se hayan presentado solicitudes de patentes.

\section{ACTIVIDAD FINANCIERA}

Nos parece obligado, en el capítulo referente a la actividad financiera, referirnos a aquellos proyectos que por índole tienen marcado tal 
signo. En tal sentido podemos referirnos, sin perjuicio de haber sido recogidos con anterioridad en la actividad legislativa global que hemos reflejado anteriormente, a los siguientes:

Proyecto de Ley de Presupuestos Generales del Estado para 1987, tramitado por el procedimiento de urgencia, y cuya discusión en el Senado se prolongó durante seis sesiones de la Cámara, quedando recogida en los DSS n. ${ }^{\circ} 12-13-14-15$ y 16 de fechas: $15-16-17$ y 19 de diciembre de 1986 , que, como es obvio, correspondería al primer período de sesiones de la Legislatura.

Por su índole, merece, igualmente ser traido al presente apartado el proyecto de Ley sobre Fiscalidad municipal en la ordenación del tráfico urbano, cuyo debate quedó reseñado en la sesión de Senado de fecha 4 de marzo de 1987, DSS n. 21.

Proyecto de Ley sobre concesión de un crédito extraordinario por importe total de 1.997.125.969 pesetas destinado a abono de pensiones asistenciales a ancianos y enfermos incapacitados, por diferencias producidas en el año 1985, quedando recogido en el DSS n. ${ }^{\circ} 28$ de abril de 1987.

Proyecto de Ley sobre dotaciones presupuestarias para inversiones y sostenimiento de las Fuerzas armadas, recogido en el DSS n. ${ }^{\circ} 28$ de abril de 1987.

Proyecto de Ley de regulación de los planes y fondos de pensiones, siendo reflejados en el DSS $n .{ }^{\circ} 33$ de fecha 20 de marzo de 1987.

\section{ACTIVIDAD DE CONTROL SOBRE EL GOBIERNO}

Esta actividad experimentó una nutrida afluencia materizalizada en preguntas, interpelaciones y mociones de muy diversa índole, tanto en el primero, como en el segundo periodo de sesiones del Senado, tal y como prevé el Reglamento del Senado, artículos 160,170 y 174 R.S., y que serian promovidos, de manera muy dispar por los diferentes grupos parlamentarios.

Haciendo una sucinta memoria de los dos períodos de sesiones que venimos tratando, podemos agrupar la actividad de control al que fue sometido el gobierno por la Cámara, a tenor de lo siguiente: 


\subsection{Primer periodo de sesiones de la III legislatura}

Que abarcaría desde octubre de 1986 a diciembre de 1986. En la sesión Plenaria, n. ${ }^{\circ} 4$ de 14 de octubre de 1986, se plantearon en la Cámara seis preguntas al Gobierno procedentes, dos de ellas del Grupo de Coalición Popular, referentes, respectivamente, a las razones que hicieron reiterar, ante el grupo de los Diez, el deseo de España de incorporarse, cuando había sido rechazada la candidatura al Grupo de los Diez, supondría para la Hacienda Española. Las otras cuatro preguntas serían formuladas por el Grupo Mixto.

En la misma Sesión, se presentaria, tal y como prescribe el artículo 170 del mencionado R.S. una interpelación del Grupo Mixto-Agrupación de Senadores del Partido Demócrata Popular, sobre suspensión para el curso 1986-1987, del Real Decreto 2.375/85 de 18 de diciembre por el que se regulan los criterios de admisión de alumnos en centros docentes sostenidos con fondos públicos.

Asimismo fue presentada una moción por parte de Coalición Popular, sobre eliminación del Acta de Adhesión de España a la CEE, de las limitaciones establecidas a la exportación agrícola e industrial. (BOCG, Senado, Serie $1, n .^{\circ} 13$ de 25 de septiembre de 1986.)

Durante la Sesión Plenaria n. 5 de 28 de octubre de 1986, se plantearian nueve preguntas al Gobierno, de las que siete serian procedentes del Grupo de Coalición Popular, de la más variada indole, una sería presentada por el Grupo Mixto y otra por el Grupo Senadores Vascos.

Fueron de igual modo planteadas dos interpelaciones por parte del Grupo Popular, referentes respectivamente a actuaciones realizadas en el proceso de elecciones sindicales, y la otra sobre medidas a adoptar por el Gobierno en materia de industria del libro (BOCG, Senado, Serie I, n. 21 de 17 de octubre de 1986).

En la Sesión Plenaria n. ${ }^{\circ} 6$ de 29 de octubre de 1986, sería planteada una moción, por parte de Coalición Popular, sobre los territorios de Ceuta y Melilla (BOCG, Senado, Serie I, n. ${ }^{\circ} 21$ de 17 de octubre de 1986).

Durante la sesión $n .^{\circ} 7$ del 30 de octubre de 1986, y como continuación de la anterior, se plantearía de igual modo, por parte de Coalición Popular, además del debate que suscitó la moción reseñada con anterioridad, otra sobre incorporación al Plan. Hidrológico Nacional de la Construcción de la presa de Rules (BOCG, Senado, Serie I, n. ${ }^{\circ} 22$ de 17 de octubre de 1986). 
La Sesión Plenaria $n .{ }^{\circ} 8$ de 11 de noviembre de 1986 , entendería de las seis preguntas, de diversa indole, cuatro de ellas presentadas por el Grupo Coalición Popular y dos por el Grupo Mixto.

De igual modo, en dicha sesión, serían planteadas Tres interpelaciones, dos de las cuales proceden del Grupo Coalición Popular y la otra de Senadores del Partido Demócrata Popular.

La Sesión Plenaria $n .^{\circ} 9$ de 12 de noviembre de 1986, conocería el debate de las dos mociones presentadas por Agrupación de Senadores del Partido Demócrata Popular - Grupo Mixto, en ambos supuestos. (BOCG, Senado, Serie I, n. ${ }^{\circ} 25$ de 29 de octubre de 1986 y BOCG, Senado, Serie I, $\mathrm{n} .^{\circ} 26$, de 4 de noviembre de 1984 , respectivamente.)

La Sesión plenaria $n .^{\circ} 10$, de fecha 2 de diciembre de 1986 , contempló la presentación de diez preguntas, de los diferentes grupos parlamentarios y dos interpelaciones procedentes del Grupo Mixto (BOCG, Senado, Serie I, n. ${ }^{\circ} 26$, de 4 de noviembre de 1986 y BOCG, Senado, Serie I, n. ${ }^{\circ} 28$ de 18 de noviembre de 1986).

Por último, en la Sesión n. ${ }^{\circ} 11$ de 3 de diciembre de 1986, sería planteada una interpelación del Grupo Coalición Popular, en materia de régimen retributivo del personal sanitario (BOCG, Senado, Serie $1, n^{\circ} 30$ de 27 de noviembre de 1986) y una noción procedente de Senadores del Partido Demócrata Popular - Grupo Mixto, relativa a centros públicos de EGB, (BOCG, Senado, Serie I, n. ${ }^{\circ} 30$ de 27 de noviembre de 1986).

\subsection{Segundo período de sesiones de la III Legislatura}

'Que se extendería desde febrero a junio de 1987. La sesión $n .^{\circ} 19$, en que se reanudaria la actividad de la Cámara tras las vacaciones parlamentarias, incluyó en su orden del día las ocho preguntas formuladas al Gobierno por los distintos Grupos Parlamentarios, de muy dispar naturaleza; de igual modo se incluirian tres interpelaciones presentadas por el Grupo Coalición Popular, Grupo Mixto y Senadores PDP - Grupo Mixto, respectivamente.

De igual forma, se tramitaría una moción conjunta de la Agrupación Senadores PDP -Grupo Mixto en rebelión al procedimiento de elección de Alcalde en los Municipios de régimen de Concejo Abierto.

La sesión Plenaria . $^{\circ} 20$ de 3 de marzo de 1987, conocería de las catorce preguntas formuladas por los diferentes grupos parlamentarios $y$ 
de las dos interpelaciones procedentes de Coalición Popular y Senadores PDP - Grupo Mixto.

Por otra parte, la sesión $n .^{\circ} 21$ de 4 de marzo de 1987 entendería de la interpelación presentada por el Grupo Mixto sobre subvenciones por parte del Consejo Superior de Deportes.

La Sesión del Pleno $n .^{\circ} 23$ de 17 de marzo de 1987, conoció las once preguntas planteadas al Gobierno y tres interpelaciones presentadas por el Grupo Coalición Popular, PDP Grupo Mixto y PDP - Grupo Mixto, respectivamente; dichas interpelaciones versarian sobre política del gobierno en materia de juego, medidas a adoptar por el Gobierno para sustanciar la responsabilidad de gestión en que haya podido incurrir el anterior Director General del Ente Público RTVE, y problemática sobre el sector vacuno de leche, como consecuencia del ingreso de España en la CEE. Sesión Plenaria n. ${ }^{\circ} 26$ de 1 de abril de 1987.

Se presentaron dos mociones referentes a las condiciones de integración del Régimen Especial de Artistas al Régimen General (Publicada en el $B O C G$, Senado, serie I, n. 60,20 de marzo de 1987) y sobre inundaciones de la presa TOUS. (BOCG, Senado, Serie I, n. 60 , fecha 20 marzo de 1987.)

En la misma sesión se presentarian asimismo, ocho preguntas de los diferentes grupos parlamentarios y una interpelación del Grupo Convergencia y Unió sobre deterioro progresivo del medio natural por la contaminación. El Pleno $n .^{\circ} 28$ de 28 de abril de 1987 experimentó una amplia actividad en materia de control por parte de la Cámara, siendo presentadas ante la misma once preguntas y una interpelación, por parte ésta última, del Grupo Coalición Popular, en lo concerniente a situación sanitaria en España.

La sesión n. 29 de abril de 1987, continuó con dicha actividad de control, conociendo dos mociones, que serian presentadas por el Grupo Coalición Popular y PDP - Grupo Mixto respectivamente, versando cada una de ellas en materia de desarrollo económico español (BOCG, Senado, Serie I, n. ${ }^{\circ} 62$ de 24 de marzo de 1987), y relativa, la segunda, a negociación con los Gobiernos autonómicos de una ordenación del Sector lechero (BOCG, Senado, Serie I, n. ${ }^{\circ} 69$ de 3 de abril de 1987).

De igual forma fueron incluidos dos interpelaciones procedentes del PDP -Grupo Mixto y Grupo Coalición Popular.

En la sesión plenaria $n{ }^{\circ} 30$ de 12 de mayo de 1987 , se plantearon diez preguntas y dos interpelaciones; éstas últimas de procedencia del 
Grupo Mixto y de Agrupación de Senadores PDP - Grupo Mixto, respectivamente.

Serían, por otra parte, presentadas en la siguiente sesión del Pleno, n. ${ }^{\circ} 31$, de fecha 13 de mayo de 1987, dos mociones de Coalición Popular, ambas en relación con la audiencia de los Ayuntamientos en las revisiones de los valores catástrofes y sobre evaluación de rendimientos del sistema educativo. En la sesión $n .^{\circ} 32$ de fecha 19 de mayo de 1987, se plantearían cinco preguntas por los diversos grupos parlamentarios; de diversa índole.

Durante las sesiones $n{ }^{\circ} 36$ y 37 de fecha 16 y 17 de junio de 1987 , fueron formuladas siete preguntas, una interpelación, procedente del Grupo Convergencia y Unió, concerniente a gastos parejos a la investigación, instando asimismo al gobierno a explicarse sobre la manera en que piensa promover la investigación privada.

Plantearianse, asimismo dos mociones, sobre supresión de tarifas portuarias en Canarias y Baleares; $y$ sobre medidas legales para servicios mínimos aéreos, en caso de huelgas, por el Senador Macías Santona y otros, y Agrupación Partido Liberal y Grupo Mixto, respectivamente.

En último término, las sesiones $\mathrm{n}^{\circ} 38$ y 39 del Senado debatirían, en fecha 23 y 24 de junio de 1987, la interpelación presentada por el PDP Grupo Mixto, acerca de la postura del Gobierno en relación con la financiación de inversión y funcionamiento de la Universidad Pública de Navarra; así como dos mociones procedentes del Grupo Coalición Popular sobre supresión de limitación del número de convocatorias en exámenes universitarios, y de la agrupación de Senadores del Partido Liberal - Grupo Mixto sobre compañias Aéreas de Ibéria y Aviaco. 